\title{
In situ monitoring of surface reactions during atomic layer etching of silicon nitride using hydrogen plasma and fluorine radicals
}

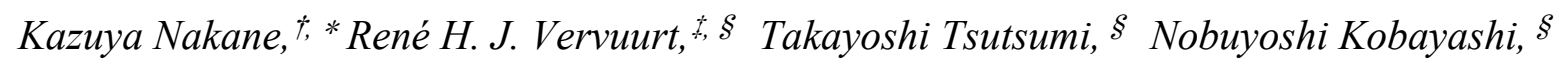

and Masaru Hori ${ }^{\S}$

${ }^{\dagger}$ Graduate School of Engineering, Nagoya University, Furo-cho, Chikusa-ku, Nagoya, Aichi 464-8603, Japan

ASM Japan K.K., 23-1, 6-chome Nagayama, Tama, Tokyo 206-0025, Japan

$\S$ Center for Low-temperature Plasma Sciences, Nagoya University, Furo-cho, Chikusa-ku, Nagoya, Aichi, 464-8603, Japan

KEYWORDS: atomic layer etching, ALE, SiN, silicon nitride, hydrogen plasma, in situ, FTIR, SE

Supporting Information Figures: 


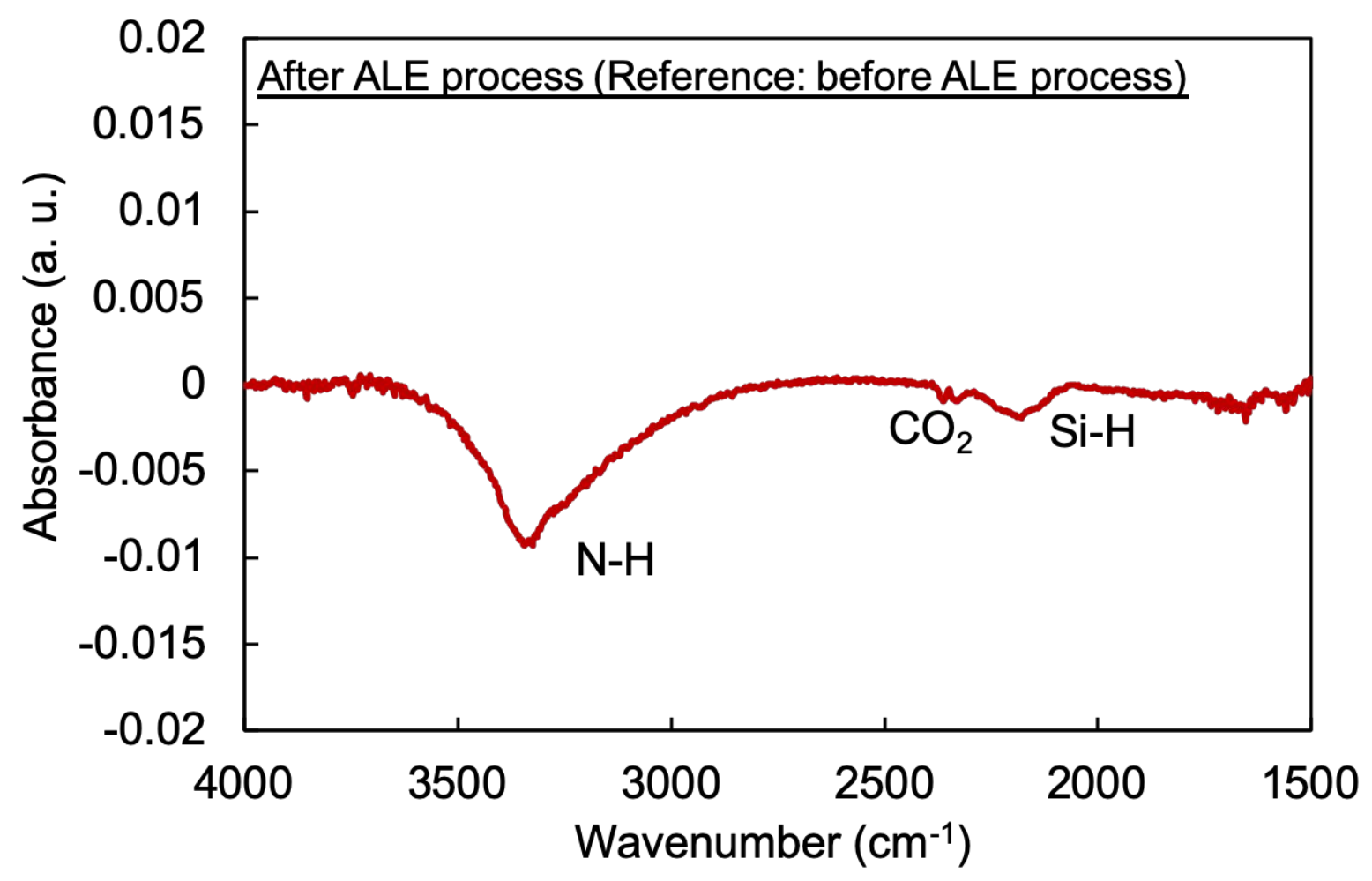

Figure S1. The in situ FTIR spectrum of SiN specimens acquired after 1 ALE cycle. The reference spectrum is that of pristine $\mathrm{SiN}$.

This figure shows the absorbance change of the SiN after one complete ALE cycle. The decrease in the absorbance in the $3500-300 \mathrm{~cm}^{-1}$ range and $2200 \mathrm{~cm}^{-1}$ indicates that there are $\mathrm{N}-\mathrm{H}$ and $\mathrm{Si}-\mathrm{H}$ bonds present in the as-deposited SiN film. 


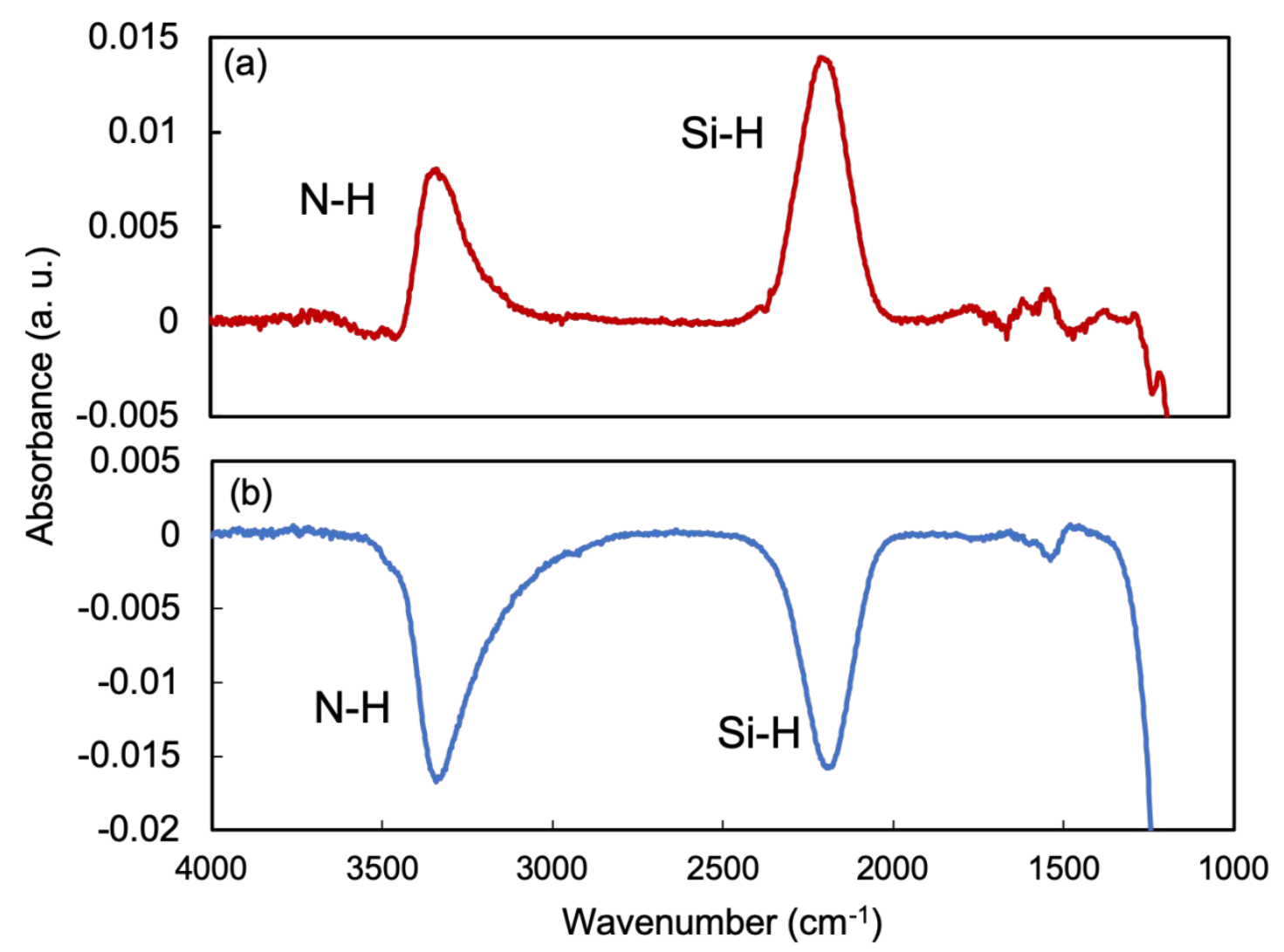

Figure S2. The in situ FTIR spectra of SiN specimens acquired (a) after hydrogen plasma exposure and (b) after fluorine radical exposure. The reference spectra are those of (a) pristine $\mathrm{SiN}$ and (b) $\mathrm{SiN}$ after exposure to hydrogen plasma for $120 \mathrm{~s}$.

These figures show the change of absorbance for each ALE half-cycle. The upper and lower spectrum show the change of hydrogen modification step and fluorine radical exposure step respectively. The $\mathrm{N}-\mathrm{H}$ and $\mathrm{Si}-\mathrm{H}$ bonds generated during hydrogen plasma modification are removed during fluorine radical exposure. 


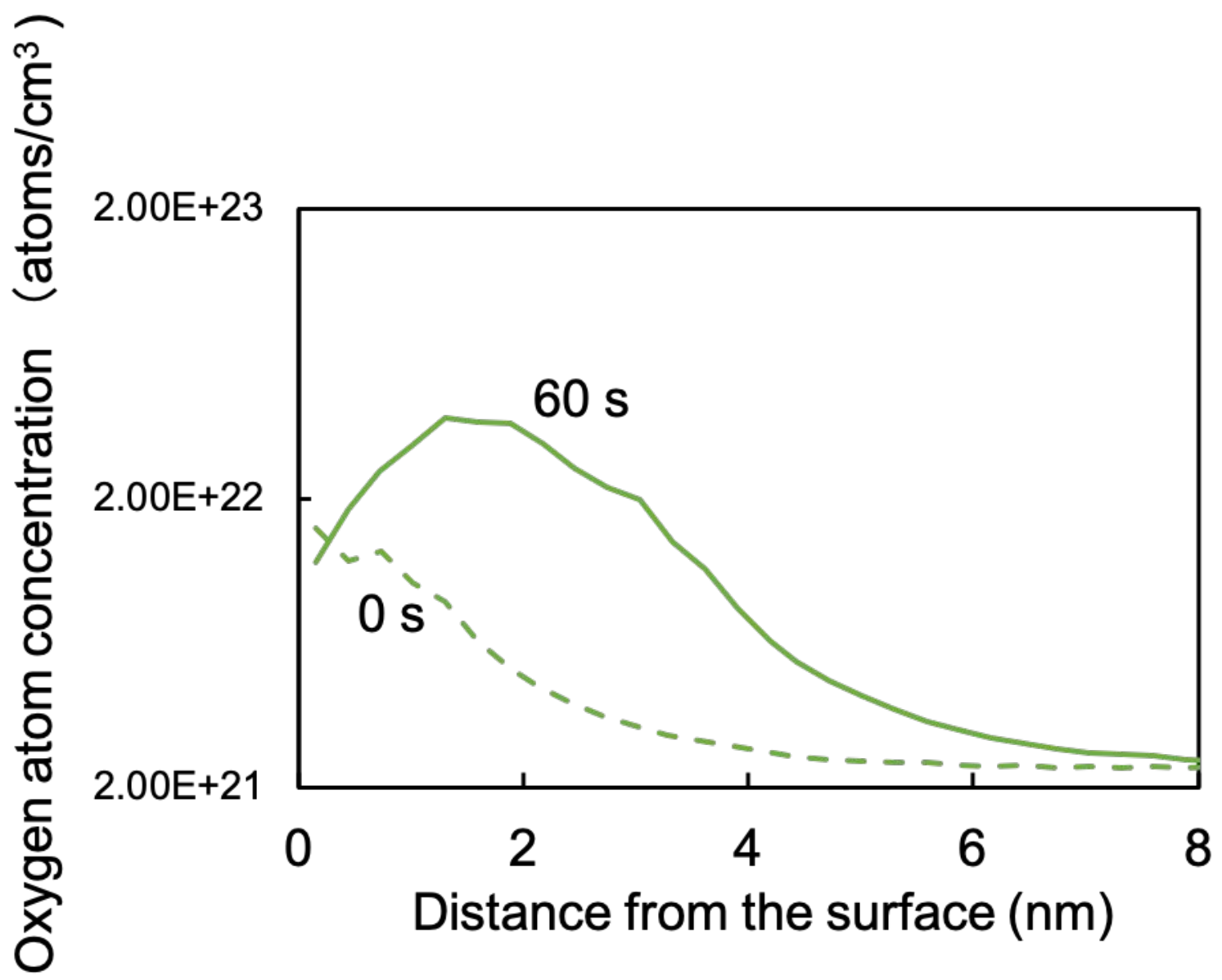

Figure S3. SIMS data showing the concentration of oxygen atoms prior to hydrogen plasma treatment and after $60 \mathrm{~s}$ hydrogen plasma exposure.

This figure presents a depth profile of the concentrations of oxygen atoms as determined using SIMS. In this figure, the results following a $60 \mathrm{~s}$ hydrogen plasma exposure without the application of bias power are compared to the results obtained before exposure to the hydrogen plasma. After $60 \mathrm{~s}$ for hydrogen exposure, the concentration of oxygen atoms increased from surface to the depth of $8 \mathrm{~nm}$. 